\title{
Research on Effects of Incidence to Turbine Guide Cascade Aerodynamic Performance
}

\author{
Zi-ming FENG, Rui SUN, Wei CUI, Jiangtao XU
}

\begin{abstract}
When steam turbine set runs, the changes of incidence angle could cause the change of the flow loss in the cascade passages. It was necessary to research the flow performance in steam turbine passages by changing incidence angle. With the help of hydrodynamic software CFX, we could conduct numerical simulations at three incidence angles of $20^{\circ}, 0^{\circ}$, and $-20^{\circ}$, respectively. The computation results indicated that the blades with aft-loading profile had a good adaptation to the incidence angle. After changing incidence angle, the incidence angle affected the distribution of static surface pressure less within most of the scope of pressure surface and suction surface than other location. However, incidence angle $-20^{\circ}$ would decrease pressure loss within a narrow range, and incidence angle $20^{\circ}$ would increase pressure loss.
\end{abstract}

Keywords: incidence angle; nozzle blade cascade; numerical study; secondary flow; steam turbine

\section{INTRODUCTION}

The steam turbine is a rotatory power machinery that can convert the energy of the steam into mechanical work. With consuming more and more non-renewable energy, more countries devote more attention to the technology of energy-saving and emission-reduction. The steam turbine consumes more energy than other mechanical engines. It is mainly used for prime mover that can generate electricity. It can also drive every kind of pump, fan, compressor and ship propeller directly. Improving efficiency of the steam turbine is one of effective methods to solve the problem of the shortage of energy resources. And enhancing overall performance of the steam turbine is the goal that every researcher works hard to achieve.

Secondary flow is the main factor that causes energy loss of steam flow in the cascades [1]. Any changes of geometric parameters or working parameters would increase the secondary flow loss as long as it could increase the transverse pressure gradient that would thicken the boundary layer or would enlarge diffuser [2, 3]. The decrease of the inlet flow angle would increase the transverse pressure in the cascades passage, the oblique cutting part produces diffuser at the entrance of cascade and produces loss under some situation. All above would increase the secondary flow. Because of the function of inlet boundary layer, the incidence angle would influence passage vortex and the core losses of main flow [4]. Generally, because of the less amount of work in the case of negative incidence angle, transverse pressure gradient was not large, and the secondary flow loss decreased. It was inverse in the case of positive incidence angle: the intensity of wall corner vortex and passage vortex was strengthened, and the passage vortex and shedding vortex moved to the center of the blade. Therefore the flow loss increased [5]. But only incidence angle was reduced within a certain range, the secondary flow could be reduced. When the incidence angle was less than a certain value, the secondary flow loss had significant growth. Yamamoto thought the reason was that in the pressure surface, flow entered into the top of the rotating cascade and went into the secondary flow [6].

In 2016, Bianchini et al. carried on a CFD simulations of NACA0018 blade profiles two airfoils generated by the NACA0018 as to compensate the virtual camber expected in VAWTs with $c / R=0.114$ and 0.25 . Numerical results were then analyzed with two novel numerical approaches, which provided proper estimations for the angle of attack on the airfoils and the relative speed in modulus and direction [7].

In 2017, Kiran et al. described the effects of nonaxisymmetric endwall profiling on the aerodynamic performance of a linear turbine cascade at different incidence angles. The calculated results indicated that the profiled endwall minimized the lateral movement of weaker boundary layer fluid from the hub-pressure surface corner. The reduction in loss coefficient was estimated to be $1.3 \%, 8.7 \%$ and $38 \%$ for incidence angles of $-10^{\circ}$, nominal and $+15^{\circ}$, respectively [8]. In 2017, Chen et al. defined two assembly error specifications of turbine blades: Circumferential Indexing Error and Axial Positioning Error, which were key factors affecting the throat and the incidence of turbine cascade. They found that the assembly error of turbine blades can be used to evaluate the aerodynamic performance and the operating safety of turbine [9]. In 2017, Xu et al. used Delayed detached eddy simulation based on Spalart-Allmaras turbulence model to investigate the flows over the threedimensional (3D) S809 airfoil at a wide range of angles of attack from $0^{\circ}$ to $90^{\circ}$. In the attached flow regime at low angle of attacks, all the simulations can give consistent results in agreement with experiment. Visualization of flows showed that $3 \mathrm{D}$ DDES can reproduce the realistic $3 \mathrm{D}$ flow structure [10].

In 2018, Wen et al. investigated the angle of attack of the blade of a horizontal offshore floating wind turbine under static and dynamic yawed conditions using the Free Vortex Method. Results showed that the angle of attack fluctuation under yawed conditions could be attributed to three effects: the blade advancing \& retreating effect, the non-uniform induction effect and the upwind \& downwind yawing effect [11]. Solis-Gallego simulated the aerodynamic field around a FX 63-137 airfoil for four angles of attack and low Reynolds numbers by CFD method with a Large Eddy Simulation (LES). The aeroacoustic calculations contribute to develop a more reliable and efficient prediction methodology based on the Computational Aeroacoustics Approach [12]. Rahimi had investigated the calculation of the angle of attack and the underlying induced velocity on wind turbine blades using data obtained from 3D-CFD. The CFD methods could be 
used to obtain airfoil characteristics for lift and drag coefficients as a function of the angle of attack [13].

In 2019, Xue et al. investigated the performance and loss mechanism of a nozzled twin entry mix-flow turbine at different admissions via experiment. Results showed that discrepancies of turbine efficiency between symmetrical unequal admissions increased when unequal admissions approached partial admissions, although their swallowing capacity was similar [14]. Elsakka et al. used a new method to calculate and store the angle of attack data during the CFD simulations without the need for extensive post-processing for efficient turbine aerodynamic analysis and optimization. The analysis illustrates how the sinusoidal variable pitch configuration could enhance the overall performance of the turbine by maintaining more favorable angle of attacks and lift and drag distributions [15].

In this paper, the experimental model was taken from the entrance guide blade cascade of a supercritical steam turbine. Using CFD technology, we researched the aerodynamic performance of different incidence angles in the inlet. The results of numerical situation showed that the $-20^{\circ}$ incidence angle could weaken pressure loss within a narrow range, and the $20^{\circ}$ incidence angle increases pressure loss.

\section{COMPUTATION SIMULATION METHOD}

\subsection{Navier-Stokes Equations}

In this simulation work, the flow media is a single-phase, isothermal, and compressible flow of gas inside the steam turbine passage.

The internal flow of steam turbine follows the rules of mass conservation equation, momentum equation, and energy conservation equation. [16]:

Mass conservation equation for flow model is as follows

$$
\frac{\partial \rho}{\partial t}+\frac{\partial(\rho u)}{\partial x}+\frac{\partial(\rho v)}{\partial y}+\frac{\partial(\rho w)}{\partial z}=0
$$

Where: $\rho$ - density $\left(\mathrm{kg} / \mathrm{m}^{3}\right) ; T-$ time $(\mathrm{s}) ; u, v$ and $w-$ velocity vector components in the $x, y$, and $z$-direction, respectively.

Momentum conservation equation for the internal flow model of the steam turbine is as follows:

Regarding the rule, the momentum conservation equation in the $x, y$ and $z$-directions can be expressed as follows:

$$
\left\{\begin{array}{l}
\frac{\partial(\rho u)}{\partial t}+\operatorname{div}(\rho u \vec{u})=-\frac{\partial p}{\partial x}+\frac{\partial \tau_{x x}}{\partial x}+\frac{\partial \tau_{y x}}{\partial y}+\frac{\partial \tau_{z x}}{\partial z}+F_{x} \\
\frac{\partial(\rho v)}{\partial t}+\operatorname{div}(\rho v \vec{u})=-\frac{\partial p}{\partial y}+\frac{\partial \tau_{x y}}{\partial x}+\frac{\partial \tau_{y y}}{\partial y}+\frac{\partial \tau_{z y}}{\partial z}+F_{y} \\
\frac{\partial(\rho w)}{\partial t}+\operatorname{div}(\rho w \vec{u})=-\frac{\partial p}{\partial z}+\frac{\partial \tau_{x z}}{\partial x}+\frac{\partial \tau_{y z}}{\partial y}+\frac{\partial \tau_{z z}}{\partial z}+F_{z}
\end{array}\right.
$$

Where: $p$ - pressure on the elemental flow volume; $\tau_{x x}, \tau_{y x}$, $\tau_{y x}$ - vector components of viscous stress; $F_{x}, F_{y}, F_{z}$ - body force on the elemental volume.

\subsection{Standard $k-\varepsilon$ Model}

In 1972, using the single equation model, Launder, and Spalding [17] proposed a two-equation model, then introduced another equation about turbulent dissipation rate $\varepsilon$, thus formed $k-\varepsilon$ model.

In the $k-\varepsilon$ model, turbulent dissipation rate $\varepsilon$ is defined as Eq. (3):

$$
\varepsilon=\frac{\mu}{\rho} \overline{\left(\frac{\partial u_{i}^{\prime}}{\partial x_{k}}\right)\left(\frac{\partial u_{j}^{\prime}}{\partial x_{k}}\right)}
$$

Here, the superscript "— " represents the average time, ""' represents the fluctuation value. Turbulent viscosity $\mu_{t}$ $\mu_{t}$ can be used to express a function of $k$ and $\varepsilon$, that is as Eq. (4):

$$
\mu_{t}=\rho C_{\mu} \frac{k^{2}}{\varepsilon}
$$

In the standard $k-\varepsilon$ model, turbulent kinetic energy $k$ and turbulent dissipation rate $\varepsilon$ are two basic unknown quantities; the corresponding transport equations were showed as Eq. (5) and Eq. (6):

$$
\begin{aligned}
& \frac{\partial}{\partial t}(\rho k)+\frac{\partial}{\partial x_{i}}\left(\rho k u_{i}\right)=\frac{\partial}{\partial x_{j}}\left[\left(\mu+\frac{\mu_{t}}{\sigma_{k}}\right) \frac{\partial k}{\partial x_{j}}\right]+ \\
& +G_{k}+G_{b}-\rho \varepsilon-Y_{M}+S_{k} \\
& \frac{\partial}{\partial t}(\rho \varepsilon)+\frac{\partial}{\partial x_{i}}\left(\rho \varepsilon u_{i}\right)=\frac{\partial}{\partial x_{j}}\left[\left(\mu+\frac{\mu_{t}}{\sigma_{\varepsilon}}\right) \frac{\partial \varepsilon}{\partial x_{j}}\right]+ \\
& +C_{1 \varepsilon} \frac{\varepsilon}{k}\left(G_{k}+C_{3 \varepsilon} G_{b}\right)-C_{2 \varepsilon} \rho \frac{\varepsilon^{2}}{k}+S_{\varepsilon}
\end{aligned}
$$

The symbols are depicted as follows: $G_{k}$ - turbulent kinetic energy due to the laminar flow velocity gradient; $G_{b}$ - because of the buoyancy induced turbulence energy $k$, for an incompressible fluid, $G_{b}=0 ; Y_{M}$ - pulsation expansion value in the compressible turbulence, for an incompressible fluid, $Y_{M}=0 ; C_{1 \varepsilon}, C_{2 \varepsilon}, C_{3 \varepsilon}$, and $C_{\mu}$ are empirical constants, according to the Launder recommended values and experimental verification in the future, $C_{1 \varepsilon}=1.44, C_{2 \varepsilon}=1.29, C_{\mu}=0.09, C_{3 \varepsilon}=0$ or $1 . C_{3 \varepsilon}$ is a buoyancy coefficient for the CFD calculation when the compressible fluid flows. When the mainstream direction parallels to the direction of gravity, $C_{3 \varepsilon}=1$. When the flow direction is perpendicular to the direction of gravity, $C_{3 \varepsilon}=$ 0 .

$\sigma_{k}$ and $\sigma_{\varepsilon}$ are corresponding to the Prandtl number of turbulent kinetic energy $k$ and dissipation rate $\varepsilon$, respectively, according to the Launder recommended values and the experimental verification, $\sigma_{k}=1.0, \sigma_{\varepsilon}=1.3$.

\section{GEOMETRY PARAMETERS, SIMULATION AND BOUNDARY CONDITIONS OF CASCADE}

Tab. 1 showed the basic geometric parameters of the prototype blade simulated by CFD. Prototype cascade 
experiments were conducted in a low-speed wind tunnel, and the working medium was air.

Table 1 Geometry parameters of cascade
\begin{tabular}{|l|l|}
\hline \multicolumn{1}{|c|}{ Parameter } & \multicolumn{1}{c|}{ Value } \\
\hline Outside diameter & $D_{\mathrm{t}}=1612 \mathrm{~mm}$ \\
\hline Middle diameter & $D_{\mathrm{m}}=1586 \mathrm{~mm}$ \\
\hline Inner diameter & $D_{\mathrm{h}}=1560 \mathrm{~mm}$ \\
\hline Blade height & $h=52 \mathrm{~mm}$ \\
\hline Chord length & $B=100 \mathrm{~mm}$ \\
\hline Pitch & $t=34.76 \mathrm{~mm}$ \\
\hline Pitch ratio & $t / b=0.35$ \\
\hline Number of blades & $N=94$ \\
\hline Geometry inlet angle & $a_{0}=90^{\circ}$ \\
\hline Geometry outlet angle & $a_{1}=15^{\circ}$ \\
\hline
\end{tabular}

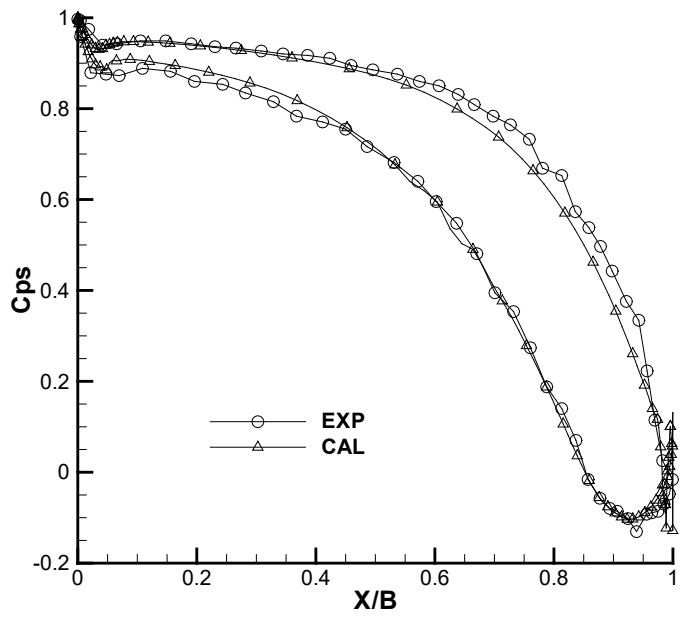

Figure 1 Surface pressure coefficient distribution along blade profile

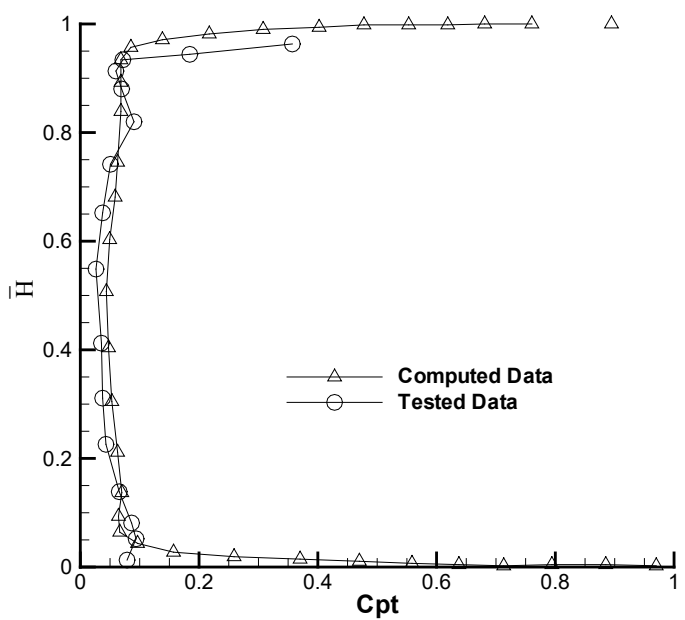

Figure 2 Pitch-average total pressure loss coefficient distributed along spanwise

Fig. 1 and Fig. 2 compare the simulation and experimental curve of static pressure coefficient and total pressure coefficient under $0^{\circ}$ incidence angle, and the results show that the error is in a reasonable range. The error is mainly caused by the deviation of calculating the incidence angle and experimental incidence angle and computing method. However, the distribution regularities of two curves are consistent basically. It does not affect the correctness of the conclusion while doing scheme contrast.

Fig. 3 depicts the tested and the simulated results of tip endwall surface streamline as the two Figures show that the numerical simulation of leading saddle point position, the tow separation lines of horseshoe vortex branch of suction surface and of pressure surface position, entering into suction surface and streamline direction, coincide very well with experimental results.

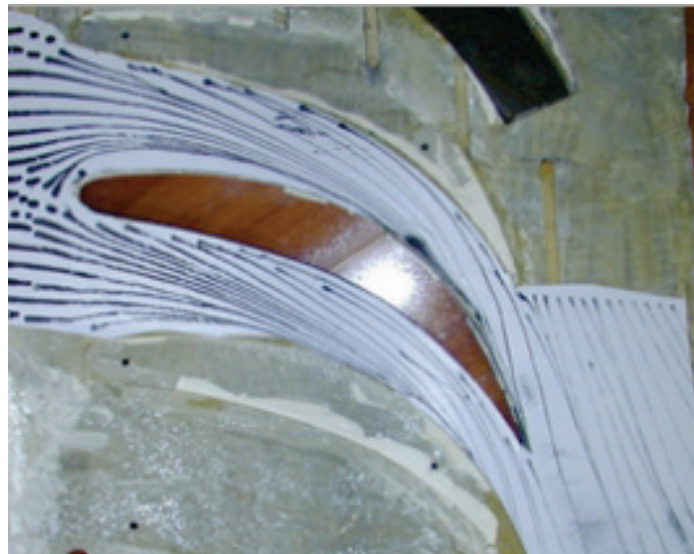

a) Test Result

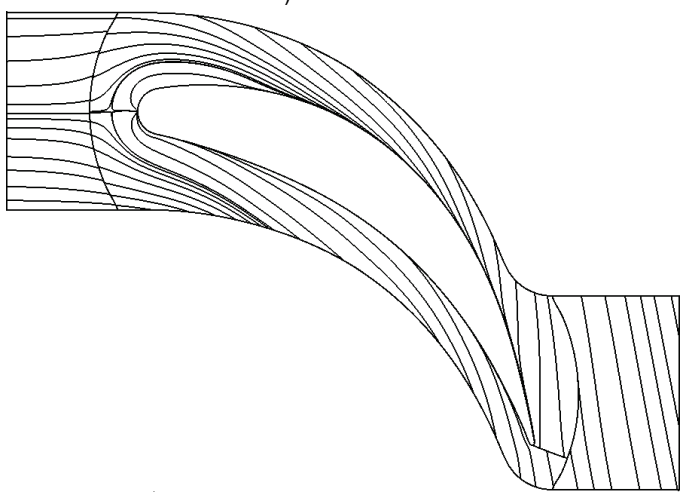

b) Computation result

Figure 3 Tip endwall streamline

Therefore, the CFX software can be used to simulate the internal flow field of turbomachinery and qualitatively and quantificationally reflected reality flow status.

The incidence angle of numerical simulation is $0^{\circ}, 20^{\circ}$, $-20^{\circ}$, respectively. CDF simulation software takes ANSYS-CFX software package. Fig. 4 is the geometric simulation model. Fig. 5 was part mesh of the leading edge and trailing edge. Fig. 6 is the total mesh model of the blade cascade passage. The orthogonality of grid is $35.25^{\circ}$. Length-width ratio is 127 . The total number of grid nodes is 395000, which satisfies the requirement of CFX for the quality of the grid. Boundary conditions: the total inlet pressure is $13100 \mathrm{~Pa}$; the total import temperature is $316 \mathrm{k}$; the export static pressure is $0.1 \mathrm{M} \mathrm{Pa}$, and the turbulence model takes $k-\varepsilon$ model.

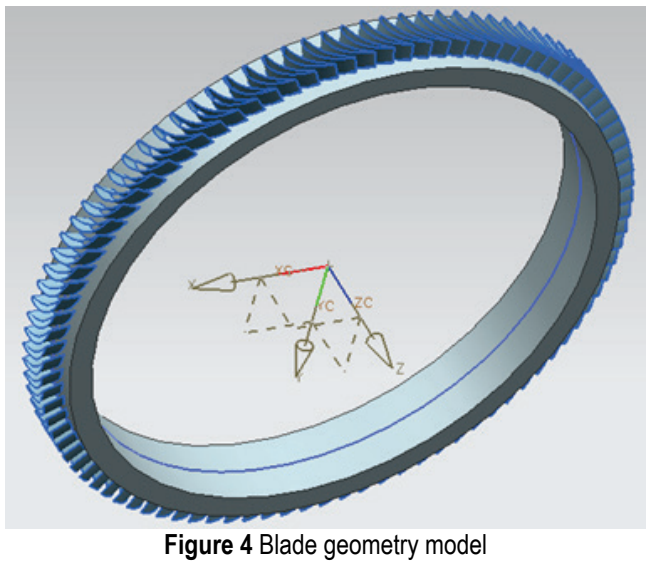




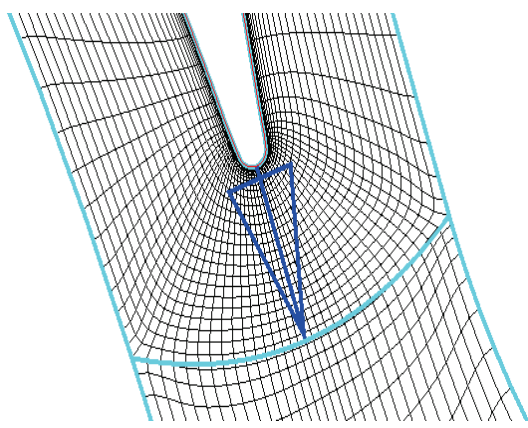

a) Part Grid of trailing edge

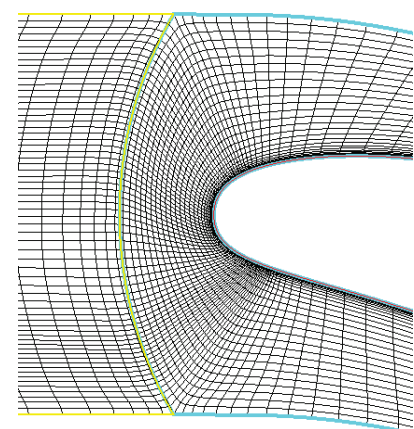

b) Part grid of leading edge

Figure 5 Part computational grid

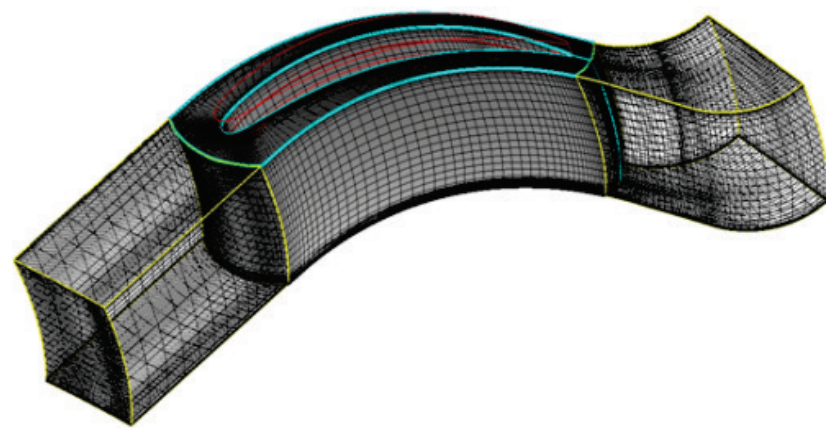

Figure 6 Flow computing grid map

\section{ANALYSIS OF CFD SIMULATION RESULTS}

\subsection{Profile Distribution of static Pressure Coefficient}

Figs. 7-9 show the static pressure coefficient distribution curves in the profile under different incidence angles, and it chose three distribution curves of static pressure coefficient which are blade root (10 percent of relatively high location), central blade (50 percent of relatively high location) and blade tip (90 percent of relatively high location). It was the aft-loading blade (The lowest pressure point of suction surface located 0.6 position after the relative axial chord length, and it is called aft-load profile) shown in Figs. 7-9, and it indicates the lowest pressure points at three incidence angles were located at 0.95 relative axial location.

Incidence angle affects the static pressure coefficient distribution in the blade profile a little. Within the relative axial scope of the blade which is less than 10 percent, under $+20^{\circ}$ and $-20^{\circ}$ incidence angle, it could form a very small period of air pressure in the blade suction surface and pressure surface. For the aft-loading blade, if incidence angle changed a little, it would not affect the static pressure coefficient distribution in blade profile very much, especially for the axial distribution of static pressure gradient. In different blade height positions, the lowest pressure point of blade suction surface was in the same position.

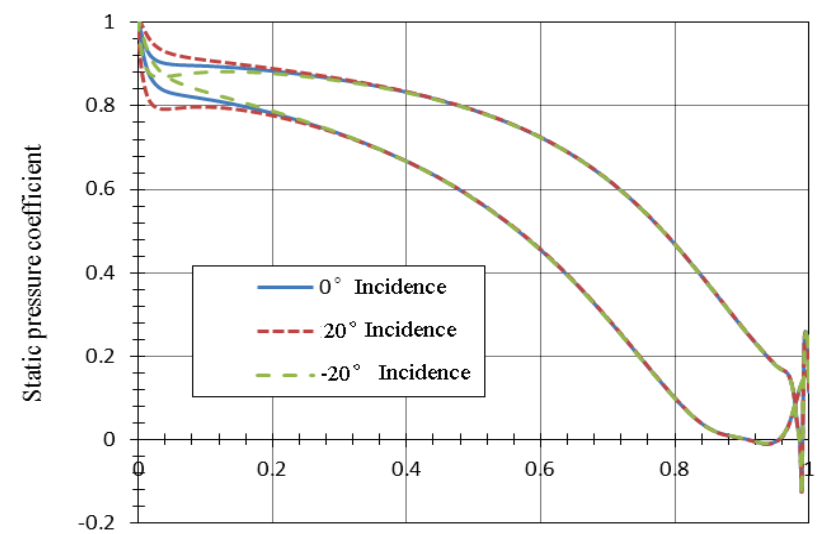

Relative axial position of blade

Figure 7 Static pressure coefficient distributed at $10 \%$ blade height

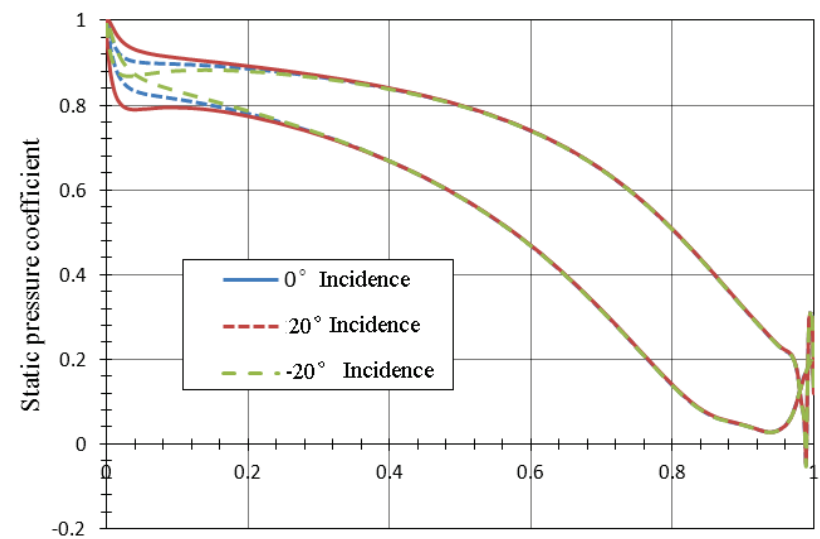

Relative axial position of blade

Figure 8 Static pressure coefficient distributed at $50 \%$ blade height

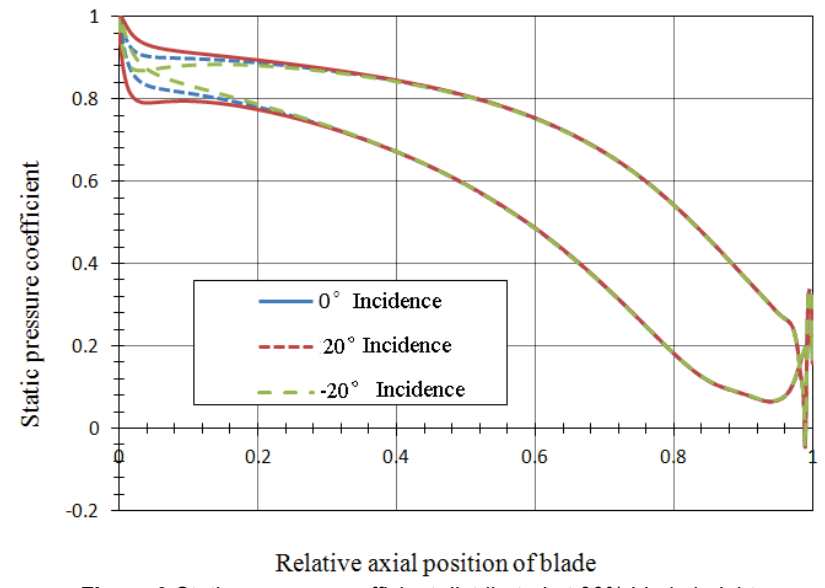

Figure 9 Static pressure coefficient distributed at $90 \%$ blade height

The area enveloped by the static pressure coefficient distribution curve in the blade profile was the load of blade as it was shown in Fig. 9. Under three incidence angles, the three static pressure coefficient curves were coincided after 0.2 times relative axial chord length location. The difference of the load of blade was within the scope of 0 0.2 times relative axial chord length where the positive incidence angle was the biggest, the $0^{\circ}$ incidence angle was the middle, and the negative incidence angle was the smallest. It was shown that when the incidence angle was 
negative, the flowing loss was the smallest, the $0^{\circ}$ incidence angle was the middle, and the positive incidence angle was the biggest.

\subsection{Profile Distribution of Meridian Plane}

This paper selected the aft-load blade as research prototype blade that had a shorter section of the adverse pressure gradient that would decrease the second flow loss in the endwall corners. The stacking line of the prototype blade was optimized to produce the radial stress that could counteract the radial pressure gradient generated by the centrifugal force produced.

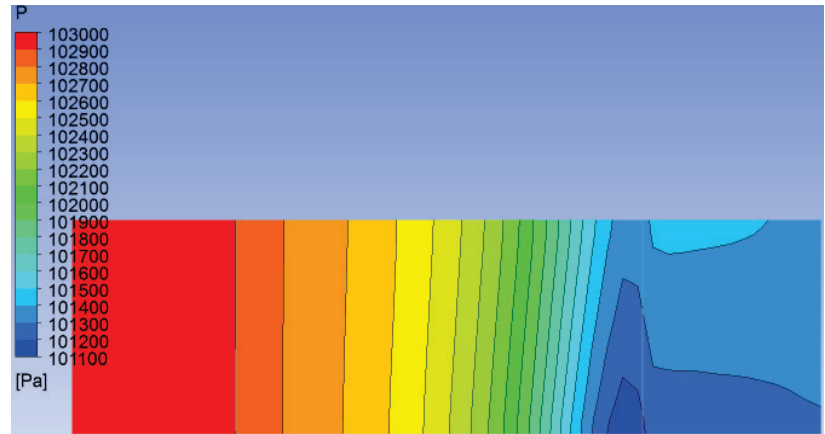

(a) Meridian plane pressure Coefficient distribution of $0^{\circ}$ incidence angle

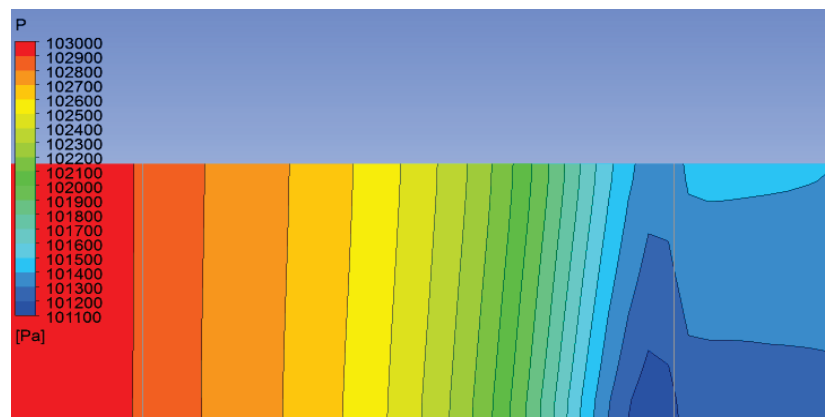

(b) Meridian plane pressure Coefficient distribution of $20^{\circ}$ incidence angle

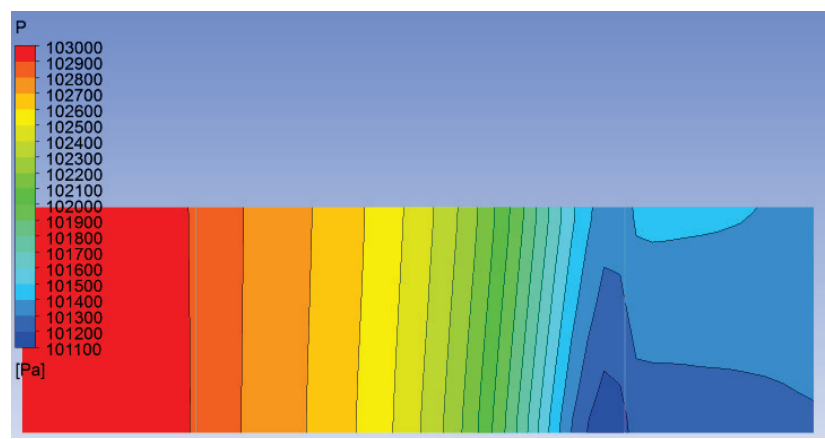

(c) Meridian plane pressure Coefficient distribution of $-20^{\circ}$ incidence angle Figure 10 Outlet total pressure loss coefficient distribution curve of three incidence angles

Therefore, the strength of concentrated vortexes (including horseshoe vortex, passage vortex, and corner vortex) was weak. The concentrated vortexes were always attached to the endwalls in the blade cascade, and the radial static pressure gradient induced by these concentrated vortexes was weak. At the three incidence angles, the distribution of static pressure isolines in the meridian plane was the same as each other. The static pressure isolines were parallel in most flowing passages. Therefore it was indicated that the main flowing in passage was laminar flowing and the loss is small. After 0.8 relative axial location, the flowing began to disorder as a result of adverse pressure gradient section with large flowing loss. Fig. 10 shows the flowing was steady at $0^{\circ}$ incidence angle, and the flowing performance was similar to the calculation results at $20^{\circ}$ and $-20^{\circ}$ incidence angles. And it was shown that the nozzle blade cascade used in this paper had a good adaptive and could run efficiently in a wide scope of the incidence angles.

\subsection{The Blade Height Distribution of the Grating Pitch Average Total Pressure Loss}

Three curves were shown in Fig. 11, blade spanwise could be divided into three areas from root to tip: root of blade, middle of blade, and tip of blade. As Fig. 11 shows, the loss of average total pressure was the smallest at $-20^{\circ}$ incidence angle, and was the largest at $20^{\circ}$ incidence angle. The total pressure loss was the largest near 0.9 and 0.1 relative height of blade that its losses core was corresponding to the passage vortex. Because of the friction loss of boundary layer, the high loss areas were near the up and down endwalls.

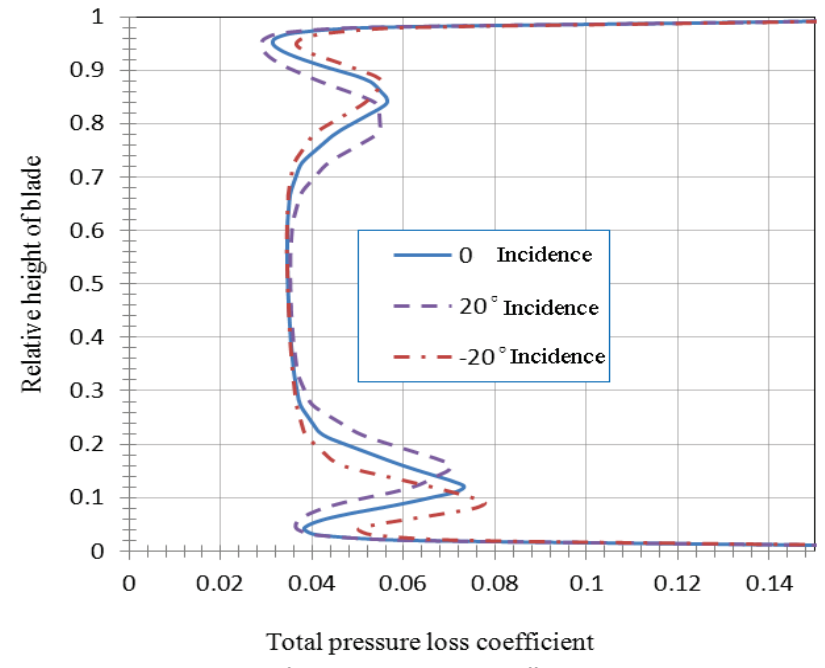

Figure 11 Distribution of total pressure loss coefficient along the spanwise

The flow loss in most of the middle spanwise was small, especially in the boundary layer of blade surface and endwalls. The boundary layer flow loss was small, but it covered most areas of the blade surface and the endwalls that indicated the total loss of boundary layer was large. The second flowing loss was caused by passage vortex and wake shedding vortex.

\subsection{The Total Pressure Isoline Distribution in the Cross- Section}

The total pressure isoline in the cross-section and in cascades passage was shown in Fig. 12. It could be seen from the total pressure isoline in the cross-section of cascades passage that the downstream flowing field had the similar total pressure distribution at the three incidence angles. That was to say that the shape of wake zones was similar, and the loss of wake zones and endwalls was large. It presented typical downstream flow structure of annular cascade. At the $8 \%$ relative location away from end walls, 
a high loss area could be found. The loss area would be enlarged with the incidence angle increasing. The width of wake flow area would be wide with the incidence angle increasing. It showed that the loss of passage vortex was the largest at $20^{\circ}$ incidence angle, middle at $0^{\circ}$ incidence, the smallest at $-20^{\circ}$ incidence angle.

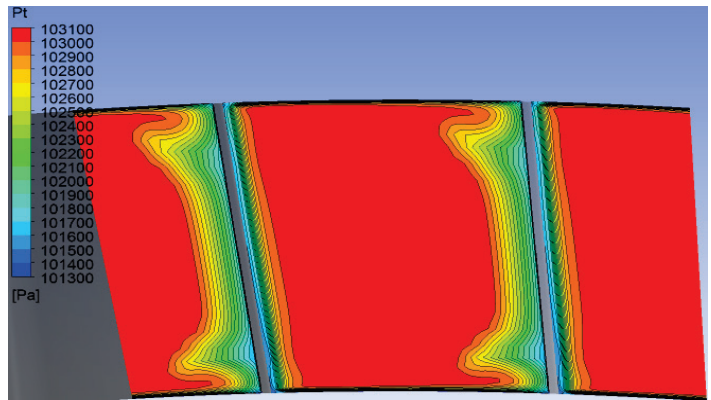

(a) $0^{\circ}$ incidence angle

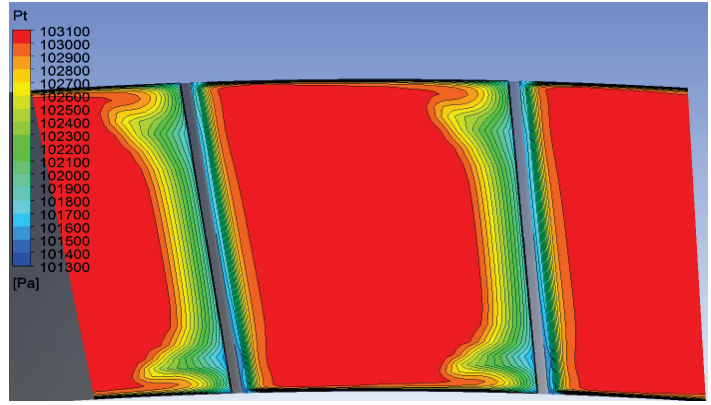

(b) $-20^{\circ}$ incidence angle

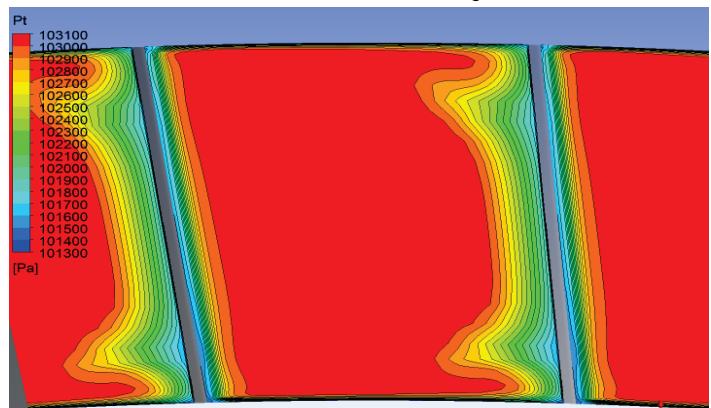

(c) $20^{\circ}$ incidence angle

Figure 12 cross-sectional isoline of the total pressure loss coefficient of aftblade

\subsection{Mach Number Distribution at Middle Section}

Fig. 13 shows the Mach number isoline distribution of three incidence angles in the middle section of passages. The annular region of Mach number isoline was located in the saddle point of horseshoe vortex. Compared with $0^{\circ}$ incidence angle, the saddle point of $-20^{\circ}$ incidence angle moved to blade suction surface, and the saddle point of $20^{\circ}$ incidence angle moved to blade pressure surface. Mach number isolines were parallel in most areas of flowing section at the three incidence angles and mostly were laminar flowing. The Mach number isoline could form enclosed area if it were near the trailing edge areas, which were three-dimensional turbulent flowing zone with larger loss. The distribution of Mach number isolines was similar at the three incidence angles. These rules above showed that the incidence angle adaptive of cascades was good.

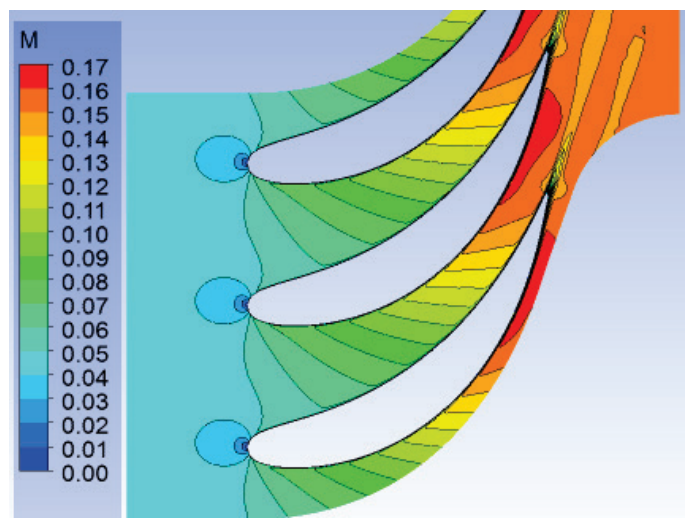

(a) $0^{\circ}$ incidence angle

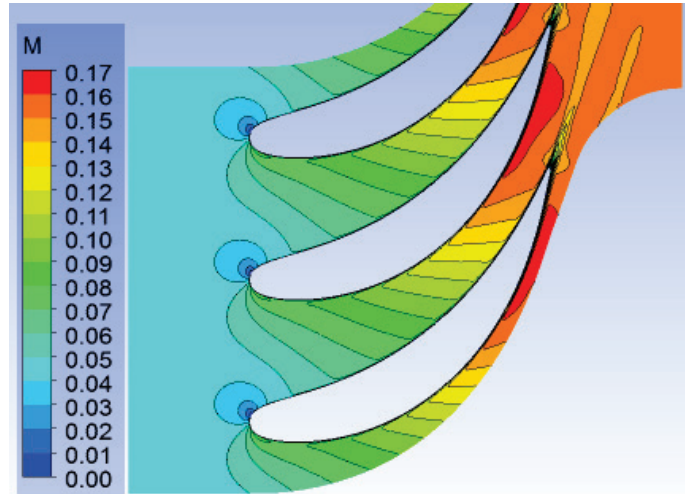

(b) $20^{\circ}$ incidence angle

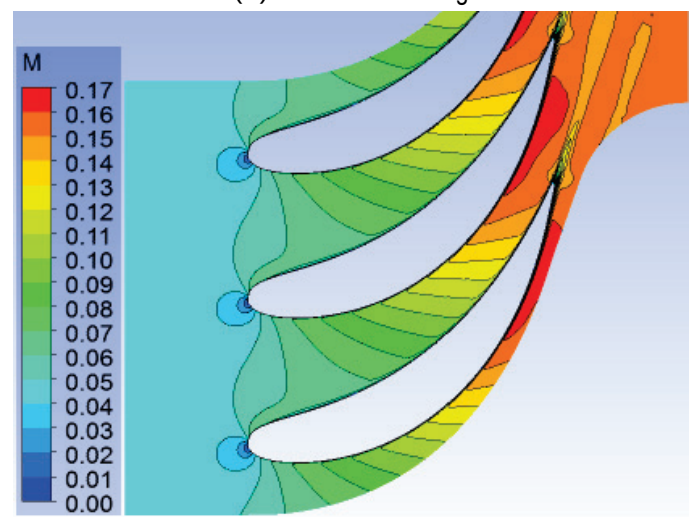

(c) $-20^{\circ}$ incidence angle

Figure 13 Mach number isoline distribution at $50 \%$ blade spanwise

\section{CONCLUSION}

The simulational blades with aft-load profile had good adaptation to the incidence angle. After changing the incidence angle, the distribution of static surface pressure would be affected less within the most scope of the pressure surface and suction surface. But at $-20^{\circ}$ incidence angle, the pressure loss within a narrow range was weakened, at $20^{\circ}$ incidence angle, the pressure loss was increased.

By analyzing the aerodynamic parameters affected by the incidence angle in each cascade flow field, no airflow jams could be found, and the up and down passage vortexes were changing with the increase of incidence angle and were enhanced. With the increase of the incidence angle, the flow loss and the width of the passage vortex increased, but the shape of the wake vortex shedding was very similar. But with the increasing of incidence angle, the flow efficiency in the cascades passage changed little. 


\section{Acknowledgments}

The project was funded by China Postdoctoral Science Foundation (No. 2018T110268; 2018M641804), Natural Science Foundation of China (No. 51774091; 51607035), Heilongjiang Postdoctoral Research Foundation (No. LBH-Q18029), Natural Science Foundation of Heilongjiang province (No. LH2019E018), Heilongjiang Youth Innovation Talents of Ordinary Undergraduate Colleges and Universities (No. UNPYSCT-2018046).

\section{Conflict of interest}

The authors declare that there are no other conflicts of interest.

\section{REFERENCES}

[1] Harvey, N. W., Martin, G. R., \& Mark, D. T. (2000). NonAxisymmetric Turbine End Wall Design: Part 1 ThreeDimensional Linear Design System. Journal of Turbomachinery, 122(2), 278-285. https://doi.org/10.1115/1.555445

[2] Wang, Z. Q. (1999). Three-Dimensional Theory and Design Method of Bowed-Twisted Blade and its Application to Turbomachines. Lecture Series - von Karman Institute for Fluid Dynamics, 2, D1-D41.

[3] Zhou, S. \& Han, W. J. (2001). Progress Review of Turbine Cascade Rectangular Whirlpool Model. Journal of Aerospace Power, 16(3), 199-202.

[4] Yamamoto, A., Tominaga, J., Matsunuma, T., \& Outa, E. (1994). Detailed Measurements of Three-Dimensional Flows and Losses Inside an Axial Turbine Rotor. ASME 1994 International Gas Turbine and Aeroengine Congress and Exposition, 94-GT-348, 1-9. https://doi.org/10.1115/94-GT-348

[5] Murty, R. G. V. \& Venkatrayulu, N. (1994). Effect of Incidence on Secondary Flows in a Linear Turbine Cascade. ASME 1994 International Gas Turbine and Aeroengine Congress and Exposition, 94-GT-381, 1-9. https://doi.org/10.1115/94-GT-381

[6] Chang, J. Z., Feng, Z. P., \& Shen, Z. D. (1998). Experimental Study of the Visual Angle of Attack Under the Cascade of Negative Secondary Flow Characteristics. Journal of Xi'an Transportation University, 32(3), 68-71.

[7] Alessandro, B., Francesco, B., Giovanni, F., \& Lorenzo, F. (2016). Virtual incidence effect on rotating airfoils in Darrieus wind turbines. Energy Conversion and Management, 111, 329-338. https://doi.org/10.1016/j.enconman.2015.12.056

[8] Kiran, K. N. \& Anish, S. (2017). An investigation on the effect of pitchwise endwall design in a turbine cascade at different incidence angles. Aerospace Science and Technology, 71, 382-391. https://doi.org/10.1016/j.ast.2017.09.032

[9] Chen, L., Li, B., \& Jiang, Z. D. (2017). Inspection of assembly error with effect on throat and incidence for turbine blades. Journal of Manufacturing Systems, 43, 366-374. https://doi.org/10.1016/j.jmsy.2017.03.007

[10] Xu, H. Y., Qiao, C. L., Yang, H. Q., \& Ye, Z. Y. (2017). Delayed detached eddy simulation of the wind turbine airfoil S809 for angles of attack up to 90 degrees. Energy, 118, 1090-1109. https://doi.org/10.1016/j.energy.2016.10.131

[11] Wen, B. R., Tian, X. L., Dong, X. J., \& Peng, Z. K. (2019). A numerical study on the angle of attack to the blade of a horizontal-axis offshore floating wind turbine under static and dynamic yawed conditions. Energy. 168, 1138-1156. https://doi.org/10.1016/j.energy.2018.11.082
[12] Solis-Gallego, I., Meana-Fernandez, A., Fernandez-Oro, J. M., Argüelles Díaz, K. M., \& Velarde-Suarez, S. (2018). LES-based numerical prediction of the trailing edge noise in a small wind turbine airfoil at different angles of attack. Renewable Energy, 120, 241-254. https://doi.org/10.1016/j.renene.2017.12.082

[13] Rahimi, H., Schepers, J. G., \& Shen, W. Z. (2018). Evaluation of different methods for determining the angle of attack on wind turbine blades with CFD results under axial inflow conditions. Renewable Energy, 125, 866-876. https://doi.org/10.1016/j.renene.2018.03.018

[14] Xue, Y. X., Yang, M. Y., Ricardo F. Martinez-Botas, R. F., Alessandro, R., \& Deng, K. Y. (2019). Loss analysis of a mix-flow turbine with nozzled twinentry volute at different admissions. Energy, 166, 775-788. https://doi.org/10.1016/j.energy.2018.10.075

[15] Elsakka, M. M., Ingham, D. B., Ma, L., \& Pourkashanian, M. (2019). CFD analysis of the angle of attack for a vertical axis wind turbine blade. Energy Conversion and Management, 182, 154-165. https://doi.org/10.1016/j.enconman.2018.12.054

[16] Versteeg, H. K. \& Malalasekera, W. (1995). An Introduction to Computational Fluid Dynamics: The Finite Volume Method, Wiley, New York.

[17] Launder, B. \& Spalding, D. (1972). Mathematical Models of Turbulence. Academic Press, Waltham.

\section{Contact information:}

\section{Zi-ming FENG}

School of Mechanical Science and Engineering, Northeast Petroleum University, Daqing high-tech development zone university street No. 99, Daqing,

Heilongjiang, China

E-mail: xueyuanfzm@163.com

\section{Rui SUN}

School of Mechanical Science and Engineering, Northeast Petroleum University, Daqing high-tech development zone university street No. 99, Daqing,

Heilongjiang Province, China

E-mail: srxnn@qq.com

Wei CUI

School of Mechanical Science and Engineering, Northeast Petroleum University, Daqing high-tech development zone university street No. 99, Daqing, Heilongiiang, China

E-mail: cuiweivv@126.com

Jiangtao XU (Corresponding Author)

College of Aerospace and Civil Engineering, Harbin Engineering University, Harbin Engineering University, Harbin, China, 163000

E-mail: hit_xit@163.com 